\title{
Co-presence of bisphosphonate-related osteonecrosis of the jaw and oral squamous cell carcinoma in a patient with postmenopausal osteoporosis: a role for chronic traumatism?
}

\begin{abstract}
Introduction: Postmenopausal osteoporosis is an important global issue. The treatment generally includes the administration of antiresorptive agents, bisphosphonate (BP) or denosumab. The most reported adverse reaction to BP is the Bisphosphonate Related Osteonecrosis of the Jaw (BRONJ). We report a case of BRONJ in an osteoporotic patient, who simultaneously revealed an oral squamous cell carcinoma (OSCC).
\end{abstract}

Case report: A 84-year-old Caucasian female referred to our Department complaining of acute pain of one month's duration on the gingival, to the right side of the mandible. The patient had a history of post-menopausal osteoporosis treated with per osalendronate for 15 years. Clinical examination surprisingly also revealed the suspect of the contemporary presence of OSCC, that was confirmed by radiological features and histopathology.

Conclusion: Unlike BRONJ or gastrointestinal damage associate to oral BP, oral ulcerations related to BP are not widely known. There is no clear evidence supporting the role of BP in the development of OSCC, but some cases of OSCC in patients taking BP are reported in literature. It would be prudent to screen and monitor these patients for all adverse reactions that could interest the oral cavity.

Keywords: osteoporosis, bisphosphonate; ONJ, oral squamous cell carcinoma, cancer, osteonecrosis, JAW, capacity, bisphosphonates, gastrointestinal, alendronate, mevalonate, osteoporotic, inhibition, thiazolidinediones
Volume 4 Issue 2 - 2017

\section{Giorgia Capocasale,' Vera Panzarella,' Antonio Lo Casto, ${ }^{2}$ Francesca Toia, ${ }^{3}$ Giuseppina Campisi' \\ 'Department of Surgical, University of Palermo, Italy \\ ${ }^{2}$ Sezione di Scienze Radiologiche, University of Palermo, Italy \\ ${ }^{3}$ Department of Surgical, University of Palermo, Italy}

Correspondence: Giuseppina Campisi, Department of Surgical, Oncological and Oral Sciences University of Palermo, Via del Vespro 129, 90127, Palermo, Italy, Tel +390 916552236 Email campisi@odonto.unipa.it

Received: December 23, 2016 | Published: February 28, 2017
Abbreviations: BMD, bone mineral density; BPS, bisphosphonates; BRONJ, bisphosphonate related osteonecrosis of the jaw; OSCC, oral squamous cell carcinoma

\section{Background}

Osteoporosis is an important global issue, particularly in postmenopausal women, predisposing them to an increased risk of fracture. Menopause is indeed associated with $a$ decrease of estrogen -mediated inhibition of bone remodeling and of bone mineral density (BMD). ${ }^{1}$ Menopause is also associated with an increase (in the burden) of coronary artery disease, diabetes mellitus, depression, cancer. Medications for these morbidities (e.g. selective serotonin reuptake inhibitors, proton pump inhibitors, thiazolidinediones, aromatase inhibitors) thought to be associated with secondary osteoporosis $^{2}$ as well. The majority of osteoporosis medications are antiresorptive agents, which reduce the rate of bone remodeling and the bone resorption. These classes of drugs include bisphosphonates and Denosumab. ${ }^{3}$ Bisphosphonates (BPs) have the capacity to bind stably to the mineral component of the bone tissue and to interact with sites of the cells involved in the physiological processes of bone remodeling and, in particular, with osteoclasts. BPs are classified in "Nitrogen-containing Bisphosphonates" NBP and non-NPB with different mechanisms of action: the firsts inhibit an enzyme that catalyzes the biosynthesis of sterols (cholesterol) from mevalonate; especially the formation of these groups isoprenyl involves the failed prenylation of several classes of proteins that play a key role in the maintenance of the cell cycle (cell morphology, cell proliferation, signal transduction). The seconds are metabolized inside of osteoclasts in molecules similar to a non-hydrolysable ATP, causing an energy deficit and the immediate death of the cell. In general, they are all well tolerated, ${ }^{4}$ but NBP toxicity to soft tissues has been known; indeed, they inhibit epithelial cell proliferation in vitro by means of inhibition of farnesyl diphosphate synthase, the same enzyme which is the target of bisphosphonates in osteoclasts.

The most known adverse reaction in the oral cavity of BPs is Bisphosphonate related Osteonecrosis of the Jaw (BRONJ). The first case of BRONJ in an osteoporotic patient was reported in $2004^{6}$ and since then, a large number of case reports have been published both related to cancer and, less frequently, non-cancer patients.

The major clinical sign of BRONJ is the presence of exposed necrotic bone in the oral cavity. Still, other clinical signs, considered "minor" (e.g. mucosal fistula, swelling, abscessed formation) also in absence of bone exposed, have been shown to be important for suspected BRONJ cases. ${ }^{7}$ Indeed, diagnostic suspect of BRONJ must be supported by radiological investigation: the most frequently used radiological investigations are, on $a$ first level, the panoramic x-ray and the intraoral x-ray and on $a$ second level the computer tomography scan (CT), both spiral CT and cone beam CT (CBCT) (second level investigation) ${ }^{8}$ Other common side effects are ulcerations of the 
upper gastrointestinal tract, predominantly reported for alendronate in its oral administration, ${ }^{9}$ especially when patients have not taken BPs as instructed (lying down within 30 minutes of taking the medication). In addition, in 2009, a US Food and Drug Administration suggested an association between bisphosphonates and risk of oesophageal cancer ${ }^{10}$ but, to date, the association between oral bisphosphonate use and upper gastrointestinal cancer has not been established. ${ }^{7}$ Only few cases have been reported on patients with oral ulcerations as side effects of BPs treatment ${ }^{11,12}$ and very few cases (only two) reported an association between oral cancer and bisphosphonate treatment. ${ }^{13,14}$ We report a case of $a$ patient with BRONJ due to an oral alendronate therapy for post-menopausal osteoporosis, diagnosed contemporary to oral squamous cell carcinoma (OSCC).

\section{Case report}

A 84-year-old Caucasian female referred to the Sector of Oral Medicine "V. Margiotta", University Policlinic "P. Giaccone" of Palermo complaining of acute pain of one month's duration on the gingiva, to the right side of the mandible. The patient had a history of osteoporosis on treatment with per os alendronate for 15 years. She also had a history of hypertension treated with calcium channel blocker. On clinical examination there was a mucosal fistula with purulent discharge (Figure 1) and a chronic ulcerative lesion (Figure 2) on the left border of the tongue. The patient was edentulous, wearing two incongruous total dentures; she did not report any teeth extractions in the previous 8 years. After obtaining an informed consent from the patient, an incisional biopsy was performed on the left border of tongue and a panoramic $\mathrm{x}$-ray and maxillary computer tomography scan (CT) was also prescribed. Histopathology confirmed a diagnosis of OSCC in the non-smoker and non-drinker patient; on the other hand, panoramic $\mathrm{x}$-ray and CT showed the presence of the sclerosis bone with a radio-transparent edge in lower right mandibular hemi-arch. Both clinical and radiological features were confirmed BRONJ, stage IIb (according with SICMF/SIPMO classification). ${ }^{8}$ She was treated with antibiotics and chlorhexidine mouth rinse, and referred to III level plastic surgery Sector for staging and treatment of OSCC and BRONJ.

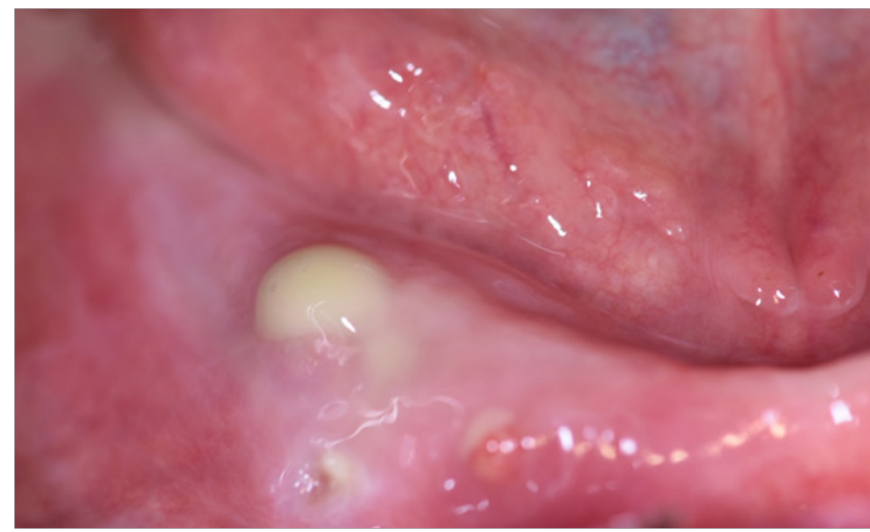

Figure I Mucosal fistula with purulent discharge.

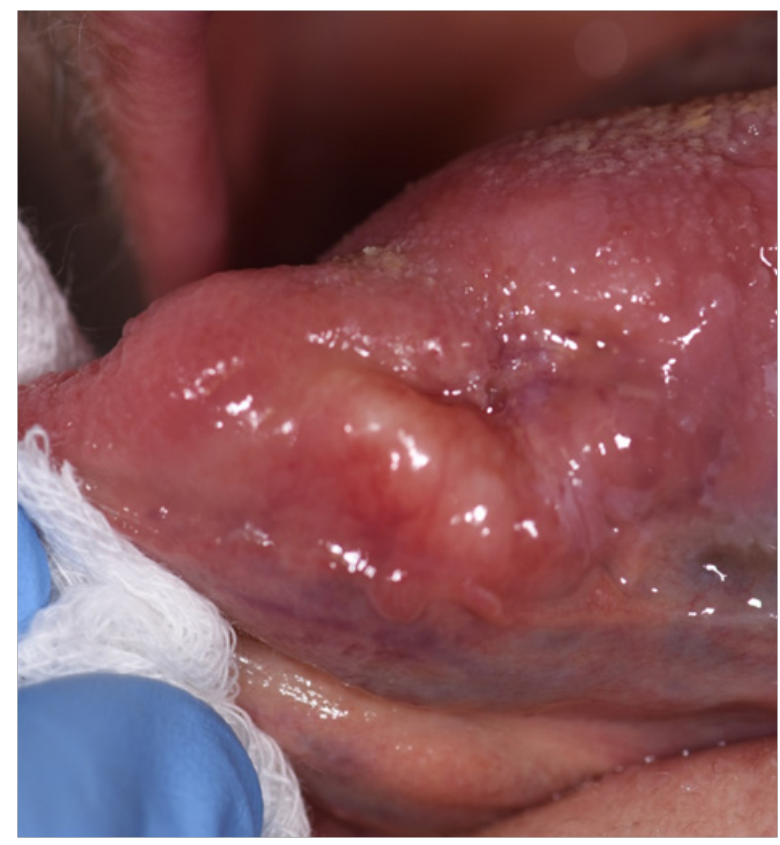

Figure $\mathbf{2}$ Chronic ulcerative lesions on the left border of tongue. 


\section{Discussion}

BRONJ is a well-known adverse reaction in cancer and, less, in non-cancer patients treated with antiresorptive agents. The diagnosis must be supported by clinical and radiological investigation. ${ }^{8}$ For patients receiving oral BPs therapy to manage osteoporosis, the prevalence of ONJ increases over time from near 0 at baseline to $0.2 \%$ after 4 or more years of BP exposure.$^{15}$ Furthermore toxicity to soft tissues has been known; in fact, this molecule inhibits epithelial cell proliferation in vitro by means of inhibition of farnesyl diphosphate synthase, the same enzyme which is the target of bisphosphonates in osteoclasts. ${ }^{5}$

Another common side effect found in literature is ulceration of the upper gastrointestinal tract, and to date, the association between oral bisphosphonate use and upper gastrointestinal cancer has been reported but, to date, not yet well-defined. ${ }^{10}$ Only few cases have been reported on patients with oral ulcerations as side effects of bisphosphonate treatment ${ }^{13,14}$ and very few cases, only two, reported an association between oral cancer and bisphosphonate treatment. ${ }^{11,12}$ Single cases do not permit to establish an association between oral BPs and OSCC, but it is suggestive to remind that in presence of local risk factors such as oral chronic traumatism (caused by incongruous dentures)oral BPs may be cause of both, ONJ and OSCC, and the patient must therefore be monitored.

\section{Authors contributions}

Giorgia Capocasale had drafted the manuscript; Vera Panzarella conceived the idea; Antonio Lo Casto had acquired and commented the imaging data, Francesca Toia helped in writing and in critical revision of the manuscript; Giuseppina Campisicarried out the supervision of the study.

\section{Conflicts of interest}

The author declares there is no conflict of interest

\section{References}

1. Management of osteoporosis in postmenopausal women: 2010 position statement of The North American Menopause Society. Menopause. 2010;17(1):25-54.

2. Mirza F, Canalis E. Secondary osteoporosis: pathophysiology and management. European Journal of endocrinology. 2015;173(3):R131R151.
3. Cosman F, Nieves JW, Dempster D. Treatment Sequence Matters: Anabolic and Antiresorptive Therapy for Osteoporosis. J Bone Miner Res. 2017;32(2):198-202.

4. Russell RG, Watts NB, Ebetino FH, et al. Mechanisms of action of bisphosphonates: similarities and differences and their potential influence on clinical efficacy. Osteoporos Int. 2008;19(6):733-759.

5. Reid IR, Bolland MJ, Grey AB. Is bisphosphonate-associated osteonecrosis of the jaw caused by soft tissue toxicity? Bone Sep. 2007;41(3):318-320.

6. Ruggiero SL, Mehrotra B. Ten years of alendronate treatment for osteoporosis in postmenopausal women. New England Journal of Medicine. 2004;351(2):191-191.

7. Campisi G, Fedele S, Fusco V, et al. Epidemiology, clinical manifestations, risk reduction and treatment strategies of jaw osteonecrosis in cancer patients exposed to antiresorptive agents. Future Oncol Feb. 2014;10(2):257-275.

8. Bedogni A, Campisi G, Fusco V. Raccomandazioni clinico-terapeutiche sull'osteonecrosi delle ossa mascellari associate a bisfosfonati e sua prevenzione. Versione Marzo. 2013;1(1).

9. Lengfeld J, Buder-Bakhaya K, Goebeler M, et al. Bisphosphonate-Mediated Oral Ulcers: A Rare Differential Diagnosis of Erosive Oral Lesions. Dermatology. 2016;232(1):117-121.

10. Vogtmann E, Corley DA, Almers LM, et al. Oral Bisphosphonate Exposure and the Risk of Upper Gastrointestinal Cancers. PLoS One. 2015;10(10):e0140180.

11. Arduino PG, Scully C, Chiusa L, et al. Oral squamous cell carcinoma arising in a patient after hematopoietic stem cell transplantation with bisphosphonate-related osteonecrosis of the jaws. Case Rep Dent. 2015;2015:1-3

12. Beattie A, Stassen L, Ekanayake K. Oral Squamous Cell Carcinoma Presenting in a Patient Receiving Adalimumab for Rheumatoid Arthritis. J Oral Maxillofac Surg Nov. 2015;73(11):2136-2141.

13. High WA, Cohen JB, Wetherington W, et al. Superficial gyrate erythema as a cutaneous reaction to alendronate for osteoporosis. $J$ Am Acad Dermatol. 2003;48:945-946.

14. Kharazmi M, Sjoqvist K, Warfvinge G. Oral ulcers, a little known adverse effect of alendronate: review of the literature. J Oral Maxillofac Surg. 2012;70:830-836.

15. Dodson TB. The Frequency of Medication-related Osteonecrosis of the Jaw and Its Associated Risk Factors. Oral Maxillofac Surg Clin N Am Nov. 2015;27(4):509-516. 